\title{
BAG6 'mops up' mislocalized proteins
}

Membrane proteins that are not correctly targeted are instead released into the cytosol. Such mislocalized proteins need to be removed to avoid, for example, the accumulation of protein aggregates. This study reveals how cells achieve this.

The authors started their search for a removal mechanism by investigating what happens to mislocalized mammalian prion protein (PrP). In cell extracts, PrP was ubiquitylated under conditions that reduce translocation, but only after it had been released into the cytosol (that is, posttranslationally). This pathway was distinct from, and faster than, other quality control pathways and depended on hydrophobic segments at the amino and carboxyl termini of $\operatorname{PrP}$, a feature of mislocalized membrane proteins that distinguishes them from bona fide cytosolic proteins.

Next, the authors looked for factors that are involved in the ubiquitylation of mislocalized proteins, identifying BAG6 as a potential candidate. BAG6, as part of a cytosolic three-protein complex, was previously found to chaperone tail-anchored membrane proteins to the endoplasmic reticulum (ER) membrane for insertion. Apparently, this ability of BAG6 to recognize tail-anchored proteins also allows interaction with the hydrophobic domains of mislocalized proteins.

To determine whether BAG6 targets mislocalized proteins for ubiquitylation, the authors depleted it from cell extracts and observed reduced ubiquitylation of many mislocalized proteins. Furthermore, control proteins that had been modified to carry hydrophobic segments depended on BAG6 for ubiquitylation. BAG6-mediated ubiquitylation was achieved through its ubiquitin-like domain, which was found to recruit the ubiquitylation machinery to the target protein.
The authors' findings suggest that, in addition to its role in targeting tail-anchored proteins to the ER, BAG6 is involved in mislocalized protein degradation. Both functions depend on the ability of BAG6 to recognize strongly hydrophobic domains on such proteins. On the basis of this, they propose that BAG6 captures ribosome-released hydrophobic proteins and acts as a quality control point, transferring only tail-anchored proteins to a targeting factor for ER insertion and holding onto the rest, which are then ubiquitylated and degraded. This fast degradation pathway may be important to ensure that cytosolic protein folding pathways are not unnecessarily occupied.

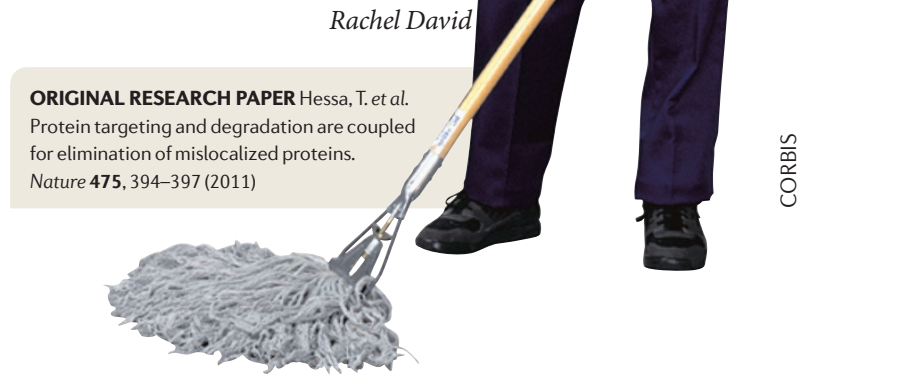

